\title{
An Ambient Intelligence System for Assisted Living
}

\author{
Alessandra De Paola, Pierluca Ferraro, Salvatore Gaglio, Giuseppe Lo Re, Marco Morana, Marco Ortolani, and \\ Daniele Peri \\ DIID Department, University of Palermo, Italy. \\ \{firstname.lastname\}@unipa.it
}

\begin{abstract}
Nowadays, the population's average age is constantly increasing, and thus the need for specialized home assistance is on the rise. Smart homes especially tailored to meet elderly and disabled people's needs can help them maintaining their autonomy, whilst ensuring their safety and well-being. This paper proposes a complete context-aware system for Ambient Assisted Living (AAL), which infers user's actions and context, analyzing its past and current behavior to detect anomalies and prevent possible emergencies. The proposed system exploits Dynamic Bayesian Networks to merge raw data coming from heterogeneous sensors and infer user's behavior and health conditions. A rule-based reasoner is able to detect and predict anomalies in such data, sending appropriate alerts to caregivers and family members. The effectiveness of the proposed AAL system is demonstrated by extensive experimental results carried out in a simulated smart home.
\end{abstract}

Keywords-Ambient Assisted Living; Multi-sensor data fusion; Dynamic Bayesian Networks; Context awareness; Rule-based Reasoning.

\section{INTRODUCTION AND RELATED WORK}

Multiple studies of the World Health Organization clearly show that the population's average age is rapidly increasing, and thus the number of elderly people is constantly growing, exceeding two billions by 2050 , according to various estimates [1]. This implies an ever increasing need for specialized home assistance. In fact, elderly people have special needs, and often have to be assisted by dedicated caregivers to live safely and comfortably.

Smart homes especially tailored to meet the needs of older people can noticeably improve their quality of life, by exploiting intelligent sensors and actuators pervasively deployed in the environment. In this regard, the paradigm of Ambient Assisted Living (AAL) [2] strives to develop unobtrusive intelligent systems that quietly assist users (and, particularly, disabled and elderly people) in their everyday life.

Recognizing users' activities is one of the key features of such systems, since it enables their modules to reason about the current situation unambiguously, thus detecting anomalies and actively taking action to prevent emergencies. In the case of patients affected by dementia, the system can even warn users if they are skipping their usual activities, such as taking medicines [3][4]. Finally, all the usual benefits of smart homes apply to elderly people as well: the system will unobtrusively modify the environment to improve their comfort and satisfy their needs, whilst reducing energy consumption [5].

Smart home systems generally exploit ambient sensors to measure physical quantities (e.g., temperature, lighting, and humidity), and actuators to automatically influence them depending on users' preferences [6]. Additionally, AAL systems leverage wearable sensors installed in smartphones, smart watches, and wristbands, to better monitor the user's behavior and disambiguate among similar situations [7]. In case of specific health conditions, it is also possible to accurately monitor patients' vital signs by means of specialized devices [8][9]. For a survey on AAL tools to improve elderly people's lives, please refer to [10]. However, accurately fusing data coming from such heterogeneous sensors can prove to be very challenging, given the intrinsic inaccuracies of sensory measurements [11]. In this regard, probabilistic techniques, such as Dynamic Bayesian Networks (DBNs) [12], can help by explicitly modeling the uncertainty of data collection. In recent years, DBNs have been exploited by many state-of-the-art systems for different applications, such as fire detection [13], target tracking [14], and user presence detection [15][16].

Furthermore, context information can be exploited by such systems to enrich raw sensor measurements, as demonstrated by many works in literature [17][18][19]. This, in turn, allows the system to better distinguish among ambiguous situations, such as a user with high temperature and blood pressure: this can be normal if the user is exercising, or anomalous if the user is not performing physical activities. Experimental results show that exploiting context information can greatly improve the system accuracy and reduce such ambiguities [20].

Selecting the best available context information is thus critical to improve the effectiveness of the system. Context information can be exploited by AAL systems as input of reasoning modules, in order to disambiguate complex situations [21]; at the same time, basic context attributes, such as time and location, may be used to refine the inference process.

Our contribution in this paper is to propose a multi-tier architecture for an AAL system that unambiguously recognizes users' behavior and health conditions, detecting potential anomalies and proactively warning caregivers and family members to prevent health emergencies and other dangerous 


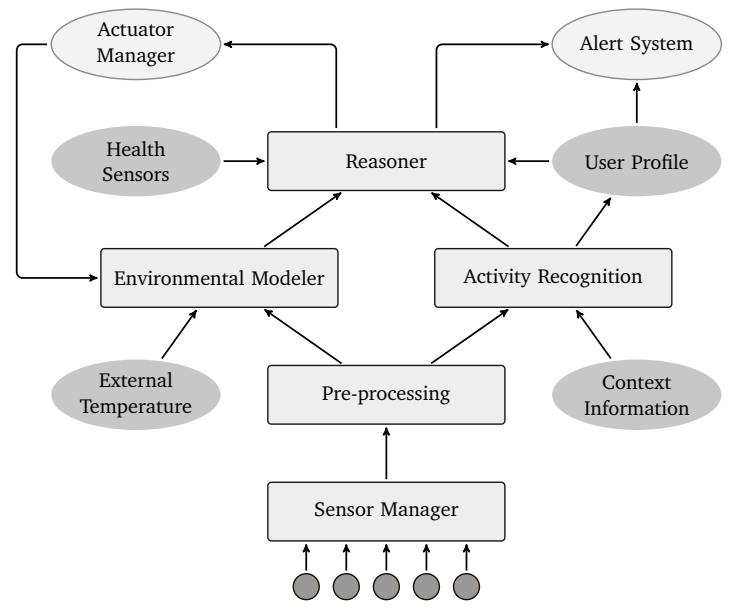

Fig. 1: Architecture of the proposed system.

situations. The end goal of such system is improving the quality of life of elderly and disabled people, by preserving their autonomy whilst ensuring their safety and maintaining their preferred environmental conditions. To this extent, we propose a context-aware, multi-sensor data fusion module based on a DBN to recognize users' activities. Such inferred information is fused with sensory readings coming from specialized health sensors, and exploited by a rule-based reasoner capable of assessing the current situation and sending alerts to family members and caregivers in case of anomalies.

The remainder of this paper is organized as follows. Section 2 presents the multi-tier architecture of our AAL system. Section 3 and Section 4 focus, respectively, on the activity recognition and rule-based reasoning modules. Section 5 describes the case study and analyzes experimental results. Finally, Section 6 presents our conclusions.

\section{Multi-Tier ArChitecture}

We propose a multi-tier architecture for an AAL system that aims to understand the surrounding environment in order to analyze the behavior of users, producing a high level description of the current context, inspired by [22]. Such awareness is exploited by a rule-based reasoner to detect and signal potential anomalies, and to actively modify the environment by controlling Heating, Ventilation, and Air Conditioning (HVAC) and lighting systems.

The proposed architecture, shown in Fig. 1, is composed by several modules. The Sensor Manager module is responsible for collecting raw data from sensors (e.g., lighting, temperature, motion and door sensors, bluetooth beacons and Microsoft Kinect devices [23]), as well as dynamically sending control messages depending on the current context, putting some of them in a low power state [24]. Collected data is then analyzed by the Pre-processing module, which filters out inaccurate readings by removing outliers and noisy measurements.

The Activity Recognition module constitutes one of the most important components of the architecture, since its accuracy heavily influences the effectiveness of the whole system. The adopted DBN exploits readily available context information and data coming from lower tiers to accurately infer the activity performed by the user and continuously update its profile.

The Environmental Modeler tries to predict the effect of actuator actions on the environment (e.g., how temperature and humidity change when turning on or off HVAC systems), so as to estimate the best course of actions to reach the desired environmental conditions. The module is trained using indoor sensors and external data coming from weather forecast stations, according to the model proposed in [25]. The parameters used by the Environmental Modeler are constantly updated by exploiting a feedback loop with the Actuator Manager and past readings from sensors, in order to maximize its prediction accuracy over time.

At the top level, the Reasoner module adopts a rule-based approach to infer the user's condition, depending on his current activity (identified by the Activity Recognition module), his vital signs (measured by specialized health sensors) and his past behavior. Depending on the estimated user's condition, the system dynamically decides whether to trigger alerts to family members and caregivers, if anomalies are detected.

Finally, the Reasoner coordinates the operations of the Actuator Manager. Such module is capable of controlling the state of the environment by means of actuators (e.g., air conditioning and lighting systems), in order to satisfy users' needs and preferences. To this end, the Reasoner plans the best sequence of actuator actions to perform in order to maintain the environmental conditions desired by the user, by exploiting the constantly updated data coming from the Environmental Modeler.

\section{ACTIVITY RECOGNITION}

In this section, we focus on the Activity Recognition module, which is fundamental for the functioning of the system. Given the unavoidable noise and inaccuracy of sensors, probabilistic data fusion techniques are often used to cope with data uncertainty. In particular, the Activity Recognition module exploits a Dynamic Bayesian Network (DBN) to better recognize dynamic phenomena, such as the users' activities.

DBNs are a generalization of Hidden Markov Models (HMMs), which allow for general topologies [12]. In a DBN, time is modeled as slices: each slice represents the state of the observed phenomena at a given moment. Each slice is composed of an arbitrary number of state nodes (e.g., the activity performed by the user) and evidence nodes (e.g., sensor readings). Our system adds the concept of context nodes, which are special evidence nodes providing further information by exploiting easily obtainable context attributes, e.g., location or time. To define a $\mathrm{DBN}$, it is necessary to specify the structure of each slice, its sensor model, and its transition model. The sensor model correlates the value of the sensory readings with that of the state variable, while the transition model specifies the probability of each possible state given its previous value.

The DBN of the Activity Recognition module maintains a belief about each possible user's activity $\left(x_{t}\right)$, in the time slice $t$, calculating the following probability:

$$
B\left(x_{t}\right)=P\left(x_{t} \mid \boldsymbol{E}_{1: t}, \boldsymbol{C}_{1: t}\right)
$$


where $\boldsymbol{E}_{1: t}$ and $\boldsymbol{C}_{1: t}$ represent, respectively, the evidences (sensor readings) and context values from the beginning to the time slice $t$. Equation (1) might seem difficult to implement in a real system, given its dependence on all previous sensor and context values. However, [24] has shown that (1) can be expressed with the following recursive equation:

$B\left(x_{t}\right)=\eta \prod_{e_{t}^{i}} P\left(e_{t}^{i} \mid\left(x_{t}\right) \cdot \sum_{x_{t-1}} P\left(x_{t} \mid x_{t-1}, \boldsymbol{C}_{t}\right) \cdot B\left(x_{t-1}\right)\right.$

where $\eta$ is a normalizing constant. Equation (2) shows that the belief can be updated recursively, knowing its value at the previous timestep $(t-1)$. It is therefore sufficient to store only two slices of the DBN at any time. In particular, [24] demonstrated that the computational complexity of calculating (2) for a single value of the state variable is $O(n+m)$, where $n$ is the number of sensor nodes, and $m$ is the number of possible values of the state variable, i.e., the number of user's activities. The overall complexity of updating the belief for each possible activity is thus $O\left(m^{2}+m \cdot n\right)$.

\section{RULE-BASED REASONER}

The Reasoning module follows a rule-based approach to determine user conditions based on information provided by the Activity Recognition module and retrieved from the user profile. Afterwards, the Reasoner uses this information to plan the actions needed to ensure user safety and comfort while also saving energy.

A special property, named user condition, heavily influences the behavior of the Reasoner module. This property describes the user's condition as it is perceived by the system, and can assume three different values, i.e., normal, anomaly or emergency. During execution, the system dynamically modifies its behavior according to the value of the user condition. For example, the Reasoner may decide to increase the sampling rate of sensors when more accuracy is needed to assess the seriousness of the situation; conversely, the system may decide to lower the sampling rate (or turn off some sensors altogether) when the condition is normal, so as to minimize energy consumption. The user condition also influences who will receive alert messages and their frequency, which can be sporadic in a normal situation or constant and very detailed in case of emergency.

In particular, the condition is considered normal if data from specialized health sensors are in the norm, and no anomalies in user behavior have been detected. In this case, the system enters into power saving mode and lowers the sampling rate of unnecessary sensors. The system reports an anomaly if the values from the health sensors are slightly out of the norm, or if it detects an inconsistency in user behavior. In this case, the Reasoning system tries to collect as much data as possible, in order to make an informed decision based on the real conditions of the user; if it deems it necessary, at this stage it starts sending alerts to family members. Finally, the system enters an emergency state if it detects a long-term anomaly or if the health data are completely out of range. The time that the system waits before considering an anomaly as long-term depends on the severity of the event itself, and can be specified by the system administrator in a fine-grained manner.
Below, we present the Prolog-like pseudo-code of a small subset of the rules used by the Reasoner, to illustrate how the system uses the user condition. The first example is a rule that puts the system in an anomaly condition if the user's blood pressure is too high and the user is lying on the bed:

anomaly_high_blood_pressure(User,UserCondition) :-

bloodPressure(high),

activity(User, lying_on_bed),

UserCondition $=$ anomaly.

It is interesting to note that the same sensory reading can also be considered perfectly normal under different circumstances. For example, if the user is doing physical activity, the system realizes this is causing high blood pressure and infers that the user's condition is not anomalous, as shown by the following rule:

normal_high_blood_pressure(User, UserCondition) :bloodPressure(high),

activity(User, exercising),

UserCondition $=$ normal.

On the other hand, if the system detects a serious medical emergency, it immediately sends warnings to family members, caregivers and medical staff:

emergency_heart_rate(User, UserCondition) :-

heartRate(very_high),

activity(User, lying_on the_floor),

UserCondition = emergency,

send_alerts([family, caregivers, medical_staff]).

We also introduce the notion of user satisfaction. When setting up the system, the user specifies his preferred temperature for each activity, along with a tolerance range and tolerance time. As a simplified example, let's assume the user's preferred temperature when working is $22{ }^{\circ} \mathrm{C}$, his tolerance range is $\pm 2{ }^{\circ} \mathrm{C}$, and his tolerance time is 10 minutes. In such a case, if the user is working and the temperature of the room is in the range $20-24{ }^{\circ} \mathrm{C}$, the user is satisfied. On the other hand, if the temperature of the room is outside this range for more than 10 minutes, he becomes unsatisfied, and directly acts to correct the temperature, by manually turning on (or off) the air conditioner.

The following rule checks whether there are satisfactory environmental conditions in the room where the user is located:

user_satisfied(User) :-

activity(User, Activity, Room),

preferred temp(User, Activity, PrefTemp),

room_temperature(Room, RoomTemp),

temp_in_range(User, RoomTemp, PrefTemp).

This rule obtains the user's inferred activity from the Activity Recognition module, interrogates the user profile to obtain his preferred temperature, checks the temperature value in the room and, finally, verifies whether the room temperature is in an acceptable range. Then, if the user is unhappy for a sufficiently long time, he becomes unsatisfied:

user_unsatisfied(User) :-

$\bar{\dagger}+$ user_satisfied(User),

unsatisfied timer(User, Timer),

timer_expired(User, Timer). 
Finally, we present one of the rules used for turning on the air conditioner if the user is dissatisfied and the room temperature is inadequate:

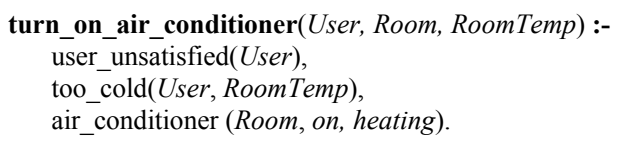

\section{EXPERIMENTAL EVALUATION}

\section{A. Experimental Setting}

We tested the effectiveness of the proposed AAL system in the application scenario of a simulated smart home. Sensor traces utilized during the experiments originate from the Aruba dataset of the Washington State University's CASAS Smart Home Project [26]. Such dataset is annotated with 11 activities of daily living (ADL), i.e., housekeeping, relax, work, sleeping, bed to toilet, meal preparation, eating, wash dishes, enter home, leave home, and resperate (a device for high blood pressure). The dataset comprises raw data collected from 39 ambient sensors (31 motion sensors, 5 temperature sensors, and 3 door sensors) over a period of seven months in a smart home with a single user. Hourly external temperature data needed by the Environmental Modeler come from a weather forecast station installed near the smart home, provided by the AgWeatherNet project of Washington State University [27].

Sensor data was preprocessed by grouping them into time windows of 30 seconds, and by adding two more ADLs, namely outside and other. The outside activity records when the user is not in the smart home (i.e., in the intervals between the leave home and enter home activities), and it is exploited by the Reasoner to infer that the majority of sensors and actuators can be safely turned off to save energy, without sacrificing accuracy and user's satisfaction.

The other activity combines all time windows that are not labeled in the original dataset. This kind of activity takes into consideration the fact that it is almost impossible to predict all activities that will be performed by users in a real setting, as suggested in [28]. Since the other activity groups heterogeneous activities, it is really difficult to classify, accounting for a $10 \%$ difference in activity recognition accuracy.

Additionally, original data have been classified based on the location of sensors, dividing the smart home into eight rooms, with an average of five sensors per room. During our experiments, we considered four main rooms, namely bedroom, living room, kitchen, and office. For the sake of simplicity, we assume that each room is provided with a 9000 BTU air conditioner, which can be automatically controlled by the Actuator Manager.

To evaluate the Activity Recognition module, we considered the average inference accuracy, defined as follows:

$$
A C C=\frac{T P+T N}{T P+T N+F P+F N}
$$

where $T P, T N, F P$, and $F N$ are the true positives, true negatives, false positives and false negatives, respectively. To
TABLE I. CROSS VALIDATION RESULTS OF THE PROPOSED SYSTEM.

\begin{tabular}{|l|l|}
\hline Accuracy & $80.5 \%$ \\
\hline Accuracy without other & $89.7 \%$ \\
\hline Uncertainty & 0.294 \\
\hline Normalized uncertainty & $7.9 \%$ \\
\hline F-score & 0.413 \\
\hline User satisfaction & $77.6 \%$ \\
\hline User satisfaction ratio & 0.917 \\
\hline Energy savings & $35.1 \%$ \\
\hline
\end{tabular}

measure inference uncertainty, we adopted an index inspired by the classical definition of Shannon's entropy [29]. Finally, we also calculated precision $(\mathrm{P})$, recall $(\mathrm{R})$, and F-score, as follows:

$$
\begin{gathered}
P=\frac{T P}{T P+F P} \quad R=\frac{T P}{T P+F N} \\
\text { F-score }=2 \cdot \frac{P \cdot R}{P+R}
\end{gathered}
$$

As explained in the previous sections, the Reasoner module leverages data coming from lower levels to infer the user's location and activity, in order to plan the best sequence of actions to ensure the user's well being. However, if the user is unhappy with any of the system's decisions, he can override them and manually modify the status of the actuators.

A workstation equipped with an Intel ${ }^{\circledR}$ Core $^{\mathrm{TM}}$ i5-3470 CPU (3.20 GHz, 4 cores, 4 GB RAM) has been used to perform all experiments.

\section{B. Experimental Results}

We adopted the cross-validation method to perform our experiments. In particular, we divided the Aruba dataset into ten parts, chose one of them as test set, and exploited the rest for training the Activity Recognition module. In the following, we will present the average results obtained by repeating our experiments ten times, with different test sets. The user condition is considered normal during all experiments.

The first set of experiments presented here evaluates the overall performance of our system, according to the metrics introduced in the previous section. To better analyze the performance of the Reasoner, we compared our smart AAL system against a baseline manual system where the user is not aided in his decisions and has to manually turn on or off the air conditioners.

Table I summarizes the results of the cross-validation experiment, reporting the average activity recognition accuracy (both considering and ignoring the other activity), the inference uncertainty (normalized and not normalized), the F-score, and the user satisfaction obtained during the simulation. Table I also reports the user satisfaction ratio, i.e., the ratio of the user satisfaction when exploiting the AAL system over the satisfaction obtained without it, and the energy savings compared to the manual system. 

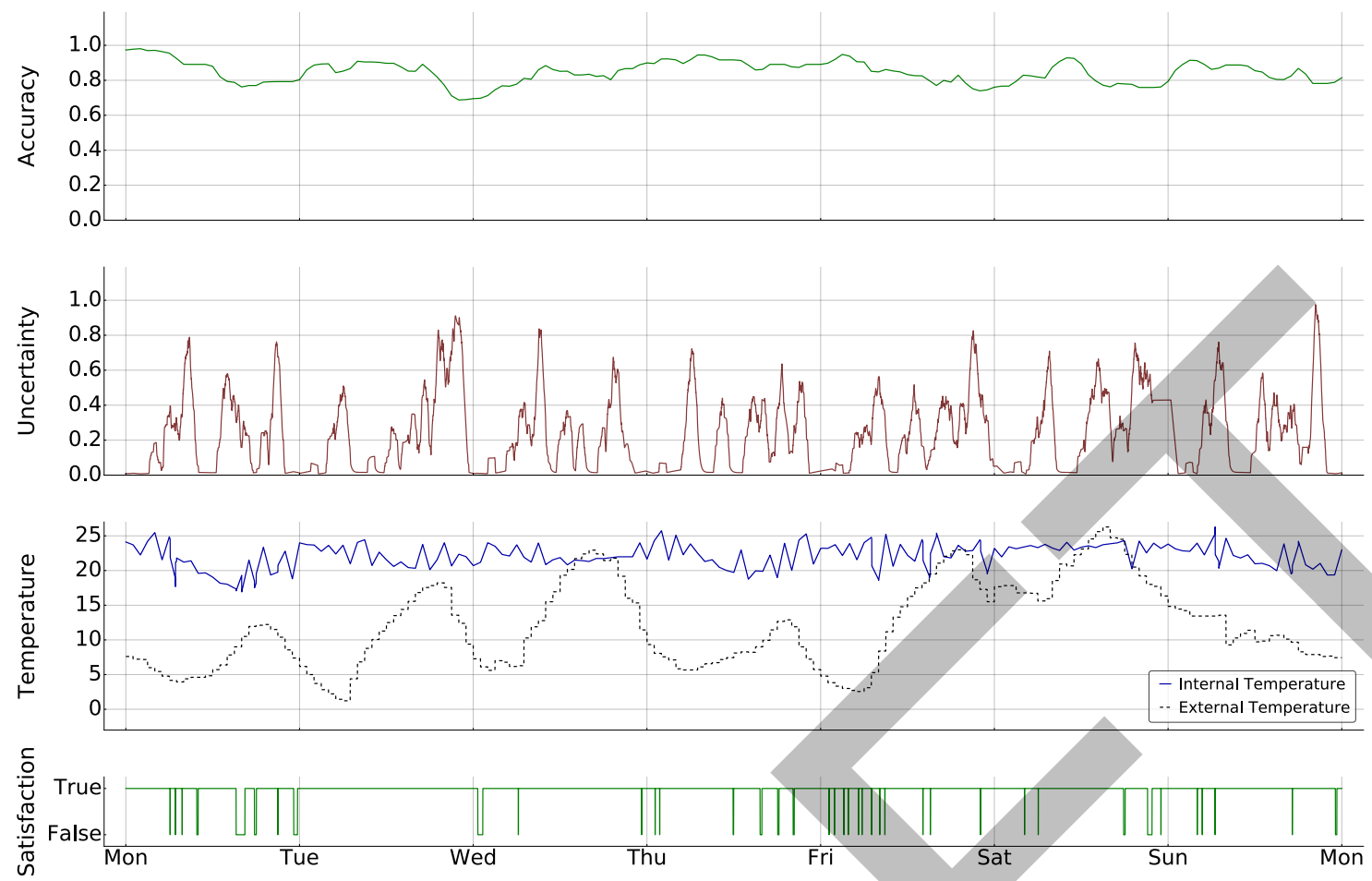

Fig. 2: Activity recognition accuracy, inference uncertainty, internal and external temperature, and user satisfaction in the living room during a given week.

We can observe that the Activity Recognition system achieves a noteworthy accuracy of $80.5 \%$, which is remarkable because it was obtained while considering the other activity. Indeed, if we repeat the experiment while ignoring such activity, the system achieves an impressive accuracy of $89.7 \%$, which is almost ten percent more. Such results are expected, considering the unpredictable nature of the other activity, which groups heterogeneous ADLs, and the peculiarity of the Aruba dataset, which mostly exploits motion sensors.

Table I also demonstrates that the system's inference uncertainty is sufficiently low throughout the experiment, reporting a value of 0.294 over a maximum of 3.7, which is the base 2 logarithm of the number of possible activities. This means that the uncertainty registered by the system is only $7.9 \%$ of the maximum. The F-score is in line with the values obtained by similar systems, as reported in [28], which compares 8 different activity recognition systems using 3 different datasets.

As regards the Reasoner module, we observe an overall $77.6 \%$ user's satisfaction, which is noteworthy if we consider that the experiment took place in a cold environment, with a minimum temperature of $-25.17{ }^{\circ} \mathrm{C}$, and that the user's preferred temperature was $22{ }^{\circ} \mathrm{C}$ on average. Filling such massive gap whilst turning off air conditioners to save energy is thus really challenging. The comparison with the manual system confirms that our AAL smart system performs very well, since the reported satisfaction ratio is 0.917 , while the average energy saving is $35.1 \%$.

Next, we present a detailed analysis of the behavior of the system in the living room. This room is ideal for our discussion, since it is the one where the user performs more activities. The first two graphs in Fig. 2 show, respectively, the accuracy and uncertainty trends. We can immediately notice that accuracy remains very high throughout the period, with an average of $84.6 \%$ and a peak of $98 \%$. At the same time, the second graph shows that uncertainty always remains below 1 , with a minimum very close to 0 .

The other two graphs in Fig. 2 concern the performance of the reasoning system. In particular, we can see how the system manages to maintain the internal temperature in the range desired by the user, with an average of $21.95^{\circ} \mathrm{C}$. Considering that the average temperature preferred by the user is $22^{\circ} \mathrm{C}$, the difference is only $0.05{ }^{\circ} \mathrm{C}$. This fact is clearly shown by the latest graph, which represents user satisfaction throughout the period. As expected, we can see that the user is almost always satisfied. This is especially good if we consider that the system is also saving more than $35 \%$ of energy with respect to a manual system, whilst keeping the user safe and satisfied. Fig. 2 also shows that the performance of the system is very regular, and is not affected by factors such as the day of the week or the period of the day, although these obviously influence the activities performed by the user.

Finally, Fig. 3 shows the activity recognition accuracy and user presence in each of the four main rooms considered in the experiments. It is interesting to note that accuracy remains very high in all rooms, which testifies to the system's excellent capabilities regardless of the environment and type of activities performed by the user. In fact, the four rooms are very different from each other. The bedroom and living room are the two areas in which the user spends the most time $34.8 \%$ and $30.8 \%$ of the time, respectively). On the contrary, kitchen and office are rarely visited ( $8 \%$ of the time in total). Nevertheless, the accuracy observed during experiments is almost the same. 


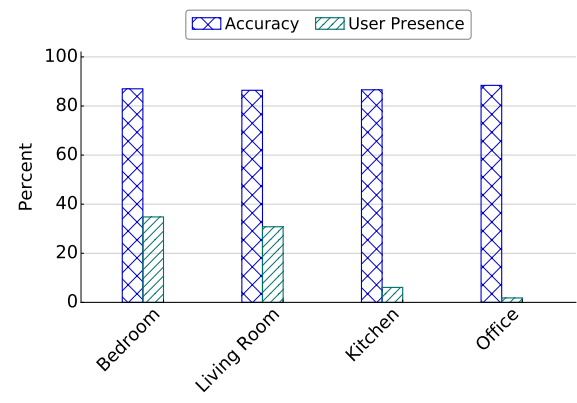

Fig. 3: Activity recognition accuracy and user presence in each room.

The bedroom and living room are also very different from each other: the first one is used almost exclusively during the night, to sleep, and therefore presents very regular and long lasting activities. Conversely, the living room offers the greatest variety of activities, as explained above. Fig. 3 therefore allows us to conclude that the system manages to maintain its high performance even when context conditions vary, which is of fundamental importance for a system that has to be used in the real world.

\section{CONCLUSIONS}

In this work, we have proposed the architecture for a complete AAL system which is capable of analyzing users' behavior and detect anomalies in their health conditions, trying to prevent emergencies by alerting appropriate caregivers and family members. The end goal of our system is improving the quality of life of elderly and disabled people, by preserving their autonomy whilst ensuring their safety. Users' activities are inferred by a probabilistic multi-sensor data fusion module which also exploits available context information, such as time and location. Such high-level data is merged with readings coming from specialized health sensors to accurately determine the user's current condition. Finally, a rule-based reasoner detects anomalous situations and sends appropriate warnings to caregivers, as well as directly modifying the environment to satisfy the user.

\section{REFERENCES}

[1] Suzman, R., Beard, J.R., Boerma, T., Chatterji, S., "Health in an ageing world - what do we know?", The Lancet 385(9967), 484-486 (2015).

[2] Rashidi, P., Mihailidis, A., "A survey on ambient-assisted living tools for older adults", IEEE Journal of Biomedical and Health Informatics 17(3), 579-590 (2013).

[3] Lotfi, A., Langensiepen, C., Mahmoud, S.M., Akhlaghinia, M.J., "Smart homes for the elderly dementia sufferers: identification and prediction of abnormal behaviour", Journal of Ambient Intelligence and humanized computing 3(3), 205-218 (2012).

[4] Roy, N., Pallapa, G., Das, S.K., "A middleware framework for ambiguous context mediation in smart healthcare application", 3rd IEEE International Conference on Wireless and Mobile Computing, Networking and Communications (WiMOB), pp. 72-79.

[5] De Paola, A., Lo Re, G., Morana, M., Ortolani, M., "Smartbuildings: an AmI system for energy efficiency", in Sustainable Internet and ICT for Sustainability (SustainIT), 2015, pp. 1-7.

[6] Khaleghi, B., Khamis, A., Karray, F.O., Razavi, S.N., "Multi- sensor data fusion: a review of the state-of-the-art", Information Fusion, vol. 14, no. 1, pp. 28-44, 2013.

[7] Cottone, P., Lo Re, G., Maida, G., Morana, M., "Motion sensors for activity recognition in an ambient-intelligence scenario", 2013 IEEE
International Conference on Pervasive Computing and Communications Workshops (PERCOM Workshops), pp. 646-651.

[8] Ni, Q., Garcia Hernando, A.B., de la Cruz, I.P., "The elderly's independent living in smart homes: A characterization of activities and sensing infrastructure survey to facilitate services development", Sensors 15(5), pp. 11312-11362 (2015).

[9] Sardini, E., Serpelloni, M., "T-shirt for vital parameter monitoring", Sensors, pp. 201-205 (2014).

[10] Rashidi, P., Mihailidis, A., "A survey on ambient-assisted living tools for older adults", IEEE Journal of Biomedical and Health Informatics 17(3), pp. 579-590 (2013).

[11] De Paola, A., La Cascia, M., Lo Re, G., Morana, M., Ortolani, M., "Mimicking biological mechanisms for sensory information fusion", Biologically Inspired Cognitive Architectures, 3, pp. 27-38.

[12] Murphy, K.P., "Dynamic Bayesian networks: representation, inference and learning", Ph.D. dissertation, University of California, (2002).

[13] Cheng, N., Wu, Q., "A decision-making method for fire detection data fusion based on Bayesian approach", 4th International Conference on Digital Manufacturing and Automation (ICDMA), pp. 21-23.

[14] Zhang, Y., Ji, Q., "Active and dynamic information fusion for multisensor systems with dynamic Bayesian networks", IEEE Transactions on Systems, Man, and Cybernetics, Part B: Cybernetics 36(2), pp. 467-472 (2006).

[15] De Paola, A., La Cascia, M., Lo Re, G., Morana, M., Ortolani, M., "User detection through multi-sensor fusion in an AmI scenario", 15th International Conference on Information Fusion, pp. 2502-2509.

[16] De Paola, A., Gaglio, S., Lo Re, G., Ortolani, M, "Multi-sensor Fusion through Adaptive Bayesian Networks", AI*IA 2011, pp. 360-371.

[17] Cho, K., Hwang, I., Kang, S., Kim, B., Lee, J., Lee, S., Park, S., Song, J., Rhee, Y., "HiCon: a hierarchical context monitoring and composition framework for next-generation context-aware services", IEEE Network 22(4), pp. 34-42 (2008)

[18] Huebscher, M.C., McCann, J.A., "Adaptive middleware for contextaware applications in smart-homes", 2nd Workshop on Middleware for Pervasive and Ad-Hoc Computing, pp. 111-116.

[19] Padovitz, A., Loke, S.W., Zaslavsky, A., Burg, B., Bartolini, C., "An approach to data fusion for context awareness", Modeling and Using Context, pp. 353-367.

[20] De Paola, A., Ferraro, P., Gaglio, S., Lo Re, G., "Context-awareness for multi-sensor data fusion in smart environments", AI*IA 2016, Advances in Artificial Intelligence, pp. 377- 391.

[21] Roy, N., Das, S.K., Julien, C., "Resolving and mediating ambiguous contexts in pervasive environments", Smart Healthcare Applications and Services: Developments and Practices, pp. 122-147 (2011).

[22] De Paola, A., Gaglio, S., Lo Re, G., Ortolani, M., "An Ambient Intelligence architecture for extracting knowledge from distributed sensors", 2nd International Conference on Interaction Sciences: Information Technology, Culture and Human, pp. 104-109.

[23] Gaglio, S., Lo Re, G., Morana, M., "Human activity recognition process using 3-D posture data", IEEE Transactions on Human-Machine Systems, 45(5), pp. 586-597 (2015).

[24] De Paola, A., Ferraro, P., Gaglio, S., Lo Re, G., Das, S., "An adaptive bayesian system for context-aware data fusion in smart environments", IEEE Transactions on Mobile Computing (2016).

[25] Gruber, P., Gwerder, M., Tödtli, J., "Predictive control for heating applications", 7th REHVA World Congress, Clima 2000.

[26] Cook, D.J., "Learning setting-generalized activity models for smart spaces", IEEE Intelligent Systems 2010(99), 1 (2010).

[27] Rasul, F., Rahman, M., Shelia, V., Hill, S.E., Islam, A., Iosiliani, O., Loyd, N., Hoogenboom, G. "Agricultural Weather Network (AgWeatherNet) Science for Society" (2015).

[28] Krishnan, N.C., Cook, D.J., "Activity recognition on streaming sensor data", Pervasive and Mobile Computing, 2012.

[29] Shannon, C.E., "A mathematical theory of communication", ACM SIGMOBILE Mobile Computing and Communications Review, vol. 5, no. 1 , pp. 3-55, 2001. 